\title{
Fast 3D surface reconstruction by unambiguous compound phase coding
}

\author{
Andrea Albarelli, Emanuele Rodolà, Samuel Rota Bulò and Andrea Torsello \\ Dipartimento di Informatica - Universitá Ca’ Foscari \\ via Torino, 155 - 30172 Venice Italy \\ http://www.dsi.unive.it
}

\begin{abstract}
Phase shift methods have proven to be very robust and accurate for photometric $3 D$ reconstruction. One problem of these approaches is the existence of ambiguities arising from the periodicity of the fringe patterns. While several techniques for disambiguation exist, all of them require the projection of a significant number of additional patterns. For instance, a global Gray coding sequence or several supplemental sinusoidal patterns of different periods are commonly used to complement the basic phase shift technique. In this paper we propose a new pattern strategy to reduce the total number of patterns projected by encoding multiple phases into a single sequence. This is obtained by mixing multiple equal-amplitude sinusoidal signals, which can be efficiently computed using inverse Fourier transformation. The initial phase for each fringe is then recovered independently through Fourier analysis and the unique projected coordinate is computed from the phase vectors using the disambiguation approach based on multiple periods fringes proposed by Lilienblum and Michaelis[6]. With respect to competing approaches, our method is simpler and requires fewer structured light patterns, thus reducing the measurement time, while retaining high level of accuracy.
\end{abstract}

\section{Introduction}

The main challenge for any triangulation-based surface reconstruction technique is the assignment of reliable correspondences between features observed by two or more different points of view. Given the central role of this problem, many and diverse strategies have been proposed in literature over the past few decades [7]. When a sparse surface reconstruction is adequate, correspondences can be searched and tracked among repeatable features readily present in the scene, such as corners or edges. Unfortunately, in general it is not possible to guarantee that the same features are extracted from each image, or that the feature density is sufficient. Hence, complementary techniques, usually based on photometric correlation, are used to obtain an approximate reconstruction of the scene depth map. Structured light systems overcome these limitations as they do not rely on natural features, but instead use projected patterns of light in order to find correspondences that are usually as dense as the pixels of each image [4]. Among these, time-multiplexing strategies such as n-ary and Gray codes, as well as hybrid approaches, are by far the most utilized [5].

Simple binary coding assigns to every pixel a codeword retrieved from the digitized sequence over time of projected black and white stripes; binary coding methods require $\log _{2}(t)$ pattern images to generate $t$ code strings. Robustness of binary codes is improved by using Gray codes, where adjacent codes differ only in one bit. Both the techniques generate unique codes along each scanline, but at the same time are limited by their low resolution due to the inherently discrete nature of the coding. Also, the large number of projected patterns does not result in an increased accuracy. Generally, this class of measurements proves to be ineffective with objects having different reflective properties (such as slick metal parts or low reflective regions), thus they must rely on the assumption of uniform albedo [5].

Phase shifting methods, on the other hand, yield higher resolutions since they are based on the projection of periodic patterns with a given spatial period. Each projected pattern is obtained by spatially shifting the preceding one of a fraction of the period, and then captured by one or more cameras. The images are then elaborated and the phase information at each pixel determined by means of M-step relationships [10]. Since the phase is distributed continuously within its period, phase shifting techniques provide subpixel accuracy and achieve high measurement spatial resolution. Furthermore, the intensity change at each pixel for subsequent patterns is relative to the underlying color and reflectance, which makes phase shift locally insensitive to texture variance to a certain degree. A major drawback is that, in it basic formulation, phase shifted structured light renders only relative phase values and thus it is ambiguous. However, when both an extended measuring range and a high resolution are required, a combined approach proves 
to be very powerful. The integration of Gray code and phase shift brings together the advantages of both, providing disambiguation and high resolution, but the number of patterns to be projected increases considerably, and each strategy introduces a source of error [6].

Other high resolution shape measurement systems include optical profilometers. Non-contact phase profilometry techniques relate each surface point to three coordinates in a frame having the $\mathrm{z}$ axis orthogonal to a reference plane, which then represents the reference for the measured height $[8,12]$. In classical phase measurement profilometry, gratings or sinusoidal patterns are projected and shifted first onto the plane and then over the object to be measured. Phase information from the deformed fringe pattern is then extracted by means of various techniques. Other, more effective profilometry techniques include the well-known Fourier Transform method [11] and other interesting derivatives $[13,3]$. Fourier-based profilometry can require as few as one or two frames for depth estimation, which makes real-time reconstruction possible. Nevertheless, in profilometric methods phase variation caused by height modulation must be limited: ambiguity of the phase limits the measurement range, allowing for gauging of smooth-shaped objects only. Moreover, noise from camera, distortion of lens, difficulties of calibration, aliasing and imperfectness of the projecting unit influence the precision of most of these techniques $[2,9]$.

Recently, some number theory based methods have been proposed for disambiguation $[14,6]$. In [6] the authors relate absolute, unambiguous phase values to projector coordinates $\xi \in \mathbb{R}$, and define $\xi(u, v)$ to be the projector coordinate at pixel $(u, v)$. Then several phase shift sequences, each with a different local period $\lambda_{i}$, are projected onto the object to be measured. A phase image is obtained for each sequence through the computation of periodic phase values $\phi_{i}(\xi) \in[0,1)$ at every pixel. In addition, the fringes of a pattern are assigned sequential natural numbers $\eta_{i}(\xi) \in \mathbb{N}$, which represent a simple counting of the fringes from left to right. A projector coordinate can then be directly obtained, for all $i=1,2, \ldots, n$, from a fringe number and a phase value:

$$
\xi=\left(\eta_{i}(\xi)+\phi_{i}(\xi)\right) \lambda_{i} .
$$

Since the only available values during measurement are $\lambda$ and $\phi$, it is clear that the system of equations becomes ambiguous as the same value of $\xi$ can be obtained for different values of $\eta_{i}$. This happens when two different projector coordinates yield the same phase values for all $i$. Therefore, the authors follow a number-theoretic approach and identify a general condition for generating unambiguous pattern sequences, by defining a maximum projector coordinate $\xi_{\max }$ up to which ambiguity can be excluded. Such a coordinate is defined as the least common multiple of relatively prime periods $\lambda_{i}$, and clearly for practical advantage it must entirely cover the projector range. An efficient method is given to calculate the fringe numbers from the ambiguous phase values at each pixel, given the local period lengths. This method takes advantage of a simple relationship between phase values and fringe numbers. Given any pair of pattern sequences, the following equivalence holds for each image pixel:

$$
\lambda_{i} \phi_{i}(u, v)-\lambda_{j} \phi_{j}(u, v)=\lambda_{j} \eta_{j}(u, v)-\lambda_{i} \eta_{i}(u, v) .
$$

This makes it possible to construct a theoretical phase difference vector beforehand, and then use it to retrieve the fringe numbers when real phase measurements become available. In addition to providing an efficient way to obtain the fringe numbers, this method allows to assign each point a reliability value related to the deviation between measured and expected values. The use of theoretical phase difference vectors makes for a powerful test, which allows to identify erroneous or weak measurements (such as mixed phase values) caused, for instance, by sharp edges, involuntary object movements and light reflections. Once the unknown fringe numbers are calculated, projector coordinates can be easily retrieved for each pattern sequence with equation 1 . The independent measurements can then be averaged to obtain a unique and absolute phase value at every pixel in an efficient way, leading to an increase in accuracy of the measurements.

The big advantages of the multi-period method is its relative simplicity and high efficiency. The phase-coded images can be directly employed in general stereo reconstruction systems, ensuring high quality and density of the code. Specifically, the lack of surface points is mainly due to occlusions and camera disparity, and measurement errors are very low thanks to the averaging and validation procedures implicit to the approach, that exclude a large percentage of errors and outliers before the actual surface reconstruction takes place. The main drawback lies in the fact that, typically, three or more pattern sequences are needed to entirely cover the projector range (typical values are 800 or 1024 projector pixels). This requires the projection of as many as three times more patterns than required with classical phase shifting.

In the next section we introduce a novel coding strategy that retains the big advantages offered by the multi-period method, but requires a significantly lower number of structured light patterns while achieving comparable levels of accuracy.

\section{Compound Phase Coding}

The main idea behind the Compound Phase Coding strategy is to project several fringe patterns in a single spatiotemporal pattern. This is obtained by encoding the phases of the fringe vector as phases of a Fourier term at different 




Figure 1. The composition of $k$ fringe patterns, plus one unknown shift signal will produce a total of $2(k+1)$ image patterns that will be projected onto the surface to be reconstructed. The shift pattern (projector scaling) accounts for the unknown value of the albedo of the surface.

frequencies. Each fringe is characterized by a different period and all of them are relatively prime. This way, once recovered the single initial phase shift for each fringe, we are able to build an unambiguous code with a numerical technique similar to the one suggested in [6].

Let $\lambda_{1}, \ldots, \lambda_{k}$ be $k$ periods and let $\xi$ be the projector coordinate at some pixel. If the periods are coprime and the projector coordinates do not exceed $\prod_{j=1}^{k} \lambda_{j}$, then we have a unique phase code for $\xi$ [6]. Namely, this code is given by the vector $\phi=\left(\begin{array}{lll}\phi_{1} & \ldots & \phi_{k}\end{array}\right) \in[0,1)^{k}$, where $\phi_{j}=(\xi$ $\left.\bmod \lambda_{j}\right) / \lambda_{j}$.

Our aim is to map $\phi$ into a signal sequence of gray-scale values that can then be measured by the cameras to obtain the unique code of each fringe. We take the hint from phase shift methods that phase encodings are more robust than amplitude codings, but extend multi-period coding by decoupling the phase used to encode the message from the frequency of the sinusoidal signal used to transport it. This is done by projecting the sum of equal-amplitude sinusoidal signals at frequencies $\frac{1}{k+1}, \frac{2}{k+1}, \ldots, \frac{k}{k+1}$, where $k$ is the number of periods necessary to encode the coordinate $x_{i}$, and by encoding the phase parameters as the phases of the corresponding sinusoidal signal.

Given a phase code $\phi \in[0,1)^{k}$, we create a $(k+1)$ dimensional complex vector $\mathbf{x} \in \mathbb{C}^{k+1}$, where

$$
x_{j}= \begin{cases}0, & \text { if } j=0, \\ e^{-2 \pi i \phi_{j}}, & \text { if } 1 \leq j \leq k .\end{cases}
$$

Here, $i=\sqrt{-1}$. Note that given $x_{j}$ for any $1 \leq j \leq k$, we can compute the phase $\phi_{j}$ as

$$
\phi_{j}=\operatorname{frac}\left(1+\frac{1}{2 \pi} \arg \left(\Im\left(x_{j}\right), \Re\left(x_{j}\right)\right)\right),
$$

for any value of $s$, where $\operatorname{frac}(\cdot)$ is the fractional part of the argument, and $\Im(z), \Re(z)$ are the imaginary and real parts of $z \in \mathbb{C}$, respectively.
Each complex number $x_{j}$ represents the amplitude and phase of a sinusoidal component with frequency $\frac{j}{k+1}$ cycles per sample. Hence we can reconstruct the intensity sequence of that coordinate by computing the Inverse Discrete Fourier Transform of $\mathbf{x}$, obtaining the vector $\mathbf{y} \in \mathbb{C}^{k+1}$, where

$$
y_{n}=\frac{1}{k+1} \sum_{j=0}^{k} x_{j} e^{2 \pi i \frac{j}{k+1} n}, \quad n=0, \ldots, k,
$$

. We can then project separately the real and imaginary part of this vector as two time sequences obtaining a single set of $2(k+1)$ patterns to be projected to uniquely encode the $x_{i}$ projector coordinate (see Figure 1).

Hence, given $\mathbf{y} \in \mathbb{C}^{k+1}$, we transform it into a real vector $\mathbf{z} \in \mathbb{R}^{2(k+1)}$, where $z_{2 j-1}=\Re\left(y_{j}\right)$ and $z_{2 j}=\Im\left(y_{j}\right)$ for $1 \leq j \leq k+1$. Afterwards we scale and shift each component of $\mathbf{z}$ in order to bound them within [0,255], obtaining the sequence of gray-scale values to project in correspondence to $\xi$. Note that by applying a shift we affect the information at frequency 0 , while by scaling, we modify the amplitudes of all frequencies, without influencing the phase values.

The acquisition process introduces an additional linear deformation on $\mathbf{z}$, which depends on the physical properties of the object being scanned. Again this does not affect the phases.

Let $\overline{\mathbf{z}} \in \mathbb{R}^{2(k+1)}$ be the acquired gray-scale values and let $\overline{\mathbf{y}} \in \mathbb{C}^{k+1}$ be its representation into a complex vector. Then, the net effect of the projector scaling and the change in the reflectivity properties of the surface is a translation and scale of the observations, i.e. the relation beween the intended signal $\mathbf{y}$ and the observed signal $\overline{\mathbf{y}}$ is

$$
\overline{\mathbf{y}}=\bar{\delta}+\bar{s} \mathbf{y}
$$

for some real values $\bar{\delta}$ and $\bar{s}$. The phase code is finally recovered from $\overline{\mathbf{y}}$ by computing the Discrete Fourier Trans- 


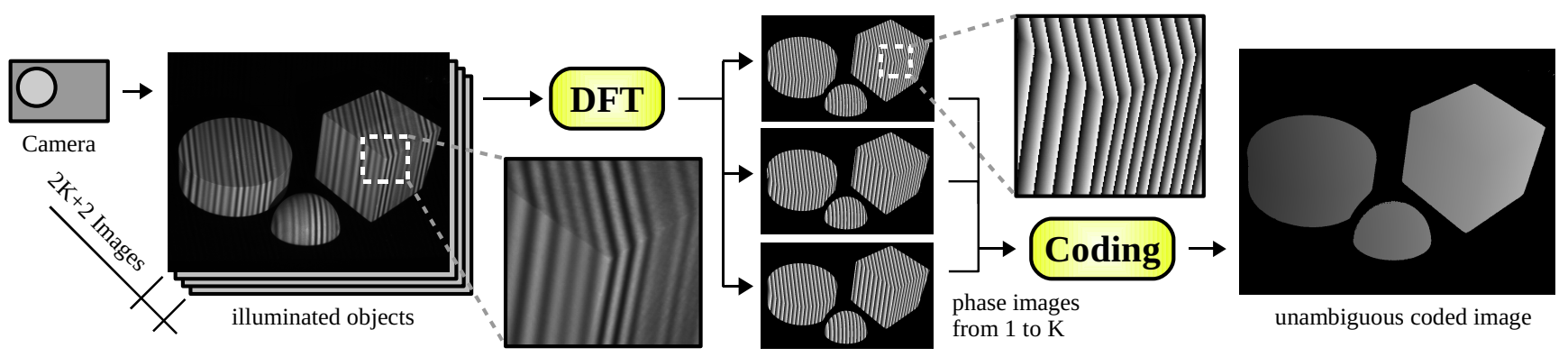

Figure 2. A total of $2 \mathrm{~K}+2$ images of illuminated objects are captured and single phase values are calculated for each composed fringe signal. Those values are subsequently used to get an unambiguous coding. Note that the intensity profile of each projected pattern is not sinusoidal.

form, namely

$$
\bar{x}_{j}=\sum_{n=0}^{k+1} \bar{y}_{n} e^{-2 \pi i \frac{n}{k+1} j},
$$

and by applying (3) to the result $\overline{\mathbf{x}} \in \mathbb{C}^{k+1}$, obtaining $\phi$ (see Figure 2).

This process allows to recover the phase code for each projector coordinate by taking only $2(k+1)$ measurements, where $k$ is the number of signal periods. Nevertheless, one can also force a larger number of samples in order to increase accuracy, by appending null components to $\mathbf{x}$. More precisely, by appending $M$ null components, we need $2(M+k+1)$ measurements in order to recall the phase code $\phi$.

It should be noted that a drawback of this approach is that encoding multiple signals in a single pattern reduces the effective projector intensity range available to encode each phase, increasing the effects of the discretization error. However, experiments show that the error introduced by this is limited.

\section{Experimental results}

In order to validate the proposed technique we need to compare its accuracy to the results obtained with a state of the art phase shift technique. To this extent we choose to compare our measurements with those given by the MultiPeriod Phase Shift proposed in [6]. The reasons for this choice are two-fold: Multi-Period Phase Shift is very accurate, as it uses information for each fringe projected in order to reduce the average error; in addition, once the phase vector from the composite signal is obtained, measurement quality is directly comparable as both techniques share the same numerical disambiguation step. In the following sections we will show both quantitative results, by evaluating the measurement error over a planar target, and qualitative results, by showing relative average distances between the measurements obtained by the two techniques with generic objects and by comparing the corresponding estimated quality.

\subsection{Experimental setup}

All the following experiments have been run on a test rig for structured light techniques that has been internally developed in our lab (figure 3). The rig is made up of a motorized plate for object positioning, four cameras and an illumination source mounted on a motorized liftable platform. Specifically the cameras are equipped with a $1 / 2$ inch CMOS sensor which offers a full 1280x1024 resolution. The cameras are monochrome, thus no Bayer filters are placed over the sensor. While four cameras are available, in this experiment set we use only one pair of cameras to reconstruct the surfaces. Thus the system falls into the category of two calibrated cameras and one uncalibrated light source. The illumination source is a 800x600 color DLP projector which we use to project the monochromatic patterns. The system is controlled by a standard PC housed into the base of the rig. This PC is a $2.8 \mathrm{GHz}$ AMD quad core system with 2 Gigabytes of ram.

Intrinsic and extrinsic parameters of the cameras have been obtained through a standard calibration procedure using a planar checkerboard and OpenCV[1] calibration soft-

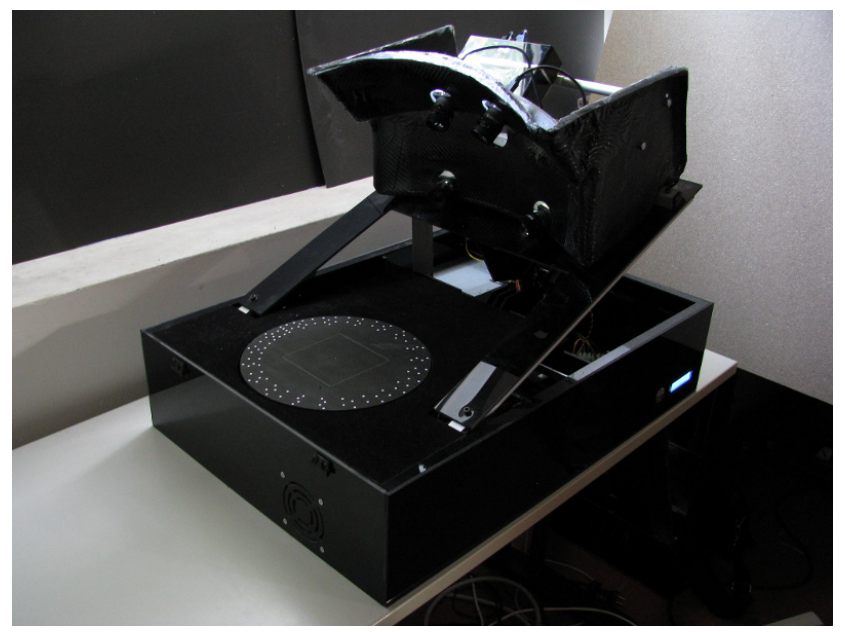

Figure 3. The general purpose structured light scanner used. 


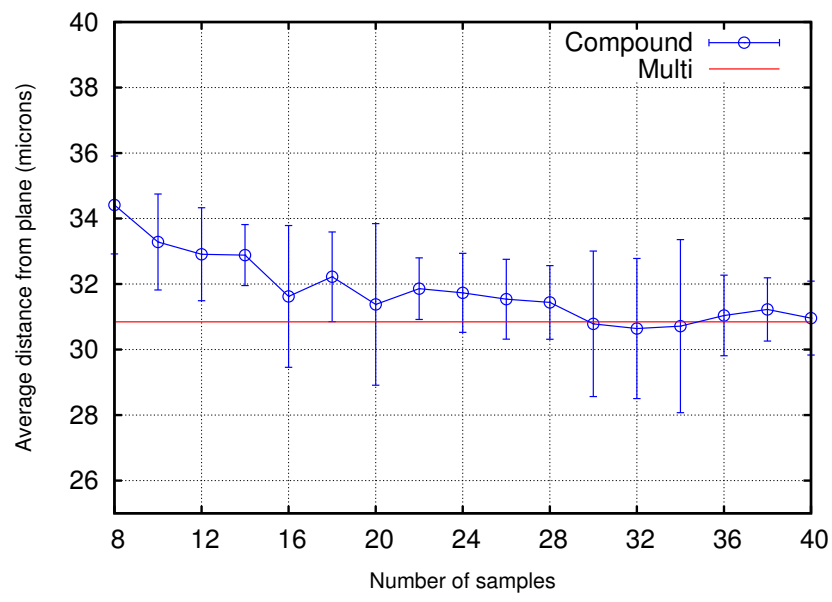

(a)

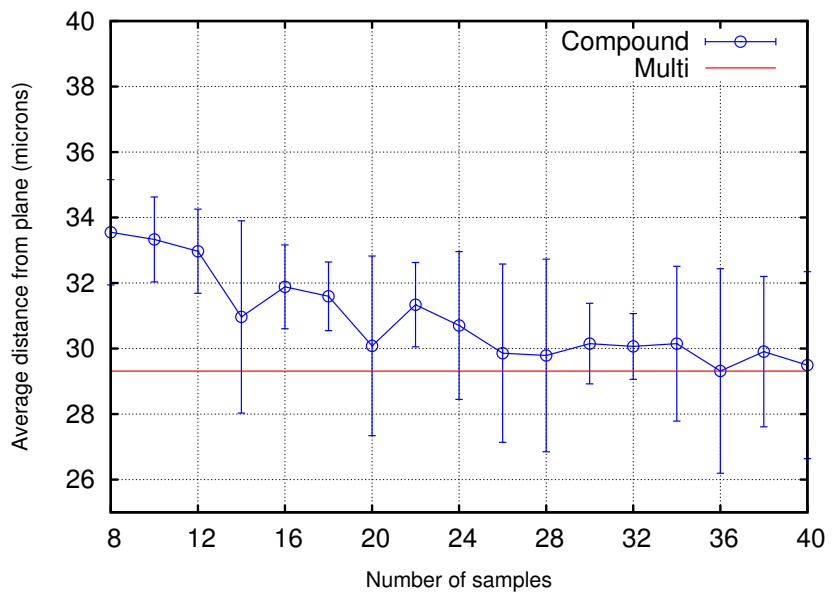

(b)

Figure 4. Accuracy comparison between the Compound Phase Coding method and the Multi-Period Phase Shift technique. In figure 4(a) we used periods of 7,11 and 13 pixels (30 patterns for Multi-Period Phase Shift), and in figure 4(b) we used periods of length 9, 11 and 13 (34 patterns for Multi-Period Phase Shift). Note that the Multi-Period technique appears as a flat continuous red line and its standard deviation as dashed red lines. Vertical bars are standard deviations in the measurement of the error for the compound technique.

ware. It must be noted that our system is not a full fledged production scanner, thus it does not guarantees extreme accuracy or resolution: in fact we estimate its precision in about $30 \mu \mathrm{m}$. This level of accuracy is adequate with respect to our experiments, as we are not interested in showing the absolute precision of our setup, but rather the relative performance of the coding schemes, showing that the proposed approach can be used as an effective replacement for slower approaches without suffering from a significant loss in accuracy.

\subsection{Planar target measurements}

In this first set of experiments we measured the surface of a 200 by $200 \mathrm{~mm}$ squared piece of float glass which we previously sprayed with a very thin layer of acrylic paint. We made several sets of measurements with both the Compound Phase Coding technique and the Multi-Period Phase Shift technique. Since the exact pose of the test object is unknown and the surface cannot be perfectly flat, we approximated the ground truth with the best fitting plane (in the least squares sense) with respect to the measured points of the object. This way we estimate the expected measurement error of each technique as the average of the absolute value of the distance from the fitting plane of each measured point. We had a wide range of choices regarding the number of different signals to project and their periods. We chose to execute the test with two configurations: 3 signals of periods respectively 7,11 and 13 pixels and other 3 signals of periods 9, 11 and 13 pixels. Since our projector has an horizontal resolution of 800 pixels both configurations allow to obtain a globally unambiguous coding of the object. Given those signal configurations, we pro- jected respectively 30 and 34 patterns for testing the MultiPeriod Phase Shift technique. Since we always used 3 signals, the Compound Phase Shift technique strictly requires only 8 patterns to be projected: nevertheless we repeated the measurement with a growing number of additional patterns in order to study the effect of the supplementary information on the final accuracy. Finally, each experimental measure was repeated for a total of 10 times. In figure 4 we compare the performance of the proposed approach against the baseline multi-period approach. Accuracy of the multi-phase approach is slightly better in 4(b), probably due to the higher number of patterns projected (34 instead of 30). The blue line shows the trend of the average error of the proposed Compound Phase Coding technique as the number of projected pattern is increased. It should be noted that even with the minimal number of projected patterns the accuracy is quite good: in fact, on average our distances from the fitting plane are only about three micrometers higher than those obtained using the Multi-Period technique, which requires almost four times as many patterns. Moreover, by projecting additional patterns, the quality of the measurements can be further enhanced: for instance, by doubling the number of projected patterns the distance from the Multi-Period approach is approximately halved. Finally, as we expected, using the same number of patterns projected by the Multi-Period technique, the two approaches yield equivalent performances. Of course, the whole point of the proposed technique is to reduce the number of projected patterns. In this sense, our approach allows to control the time/precision trade-off, obtaining good reconstructions even with just 8 patterns. 


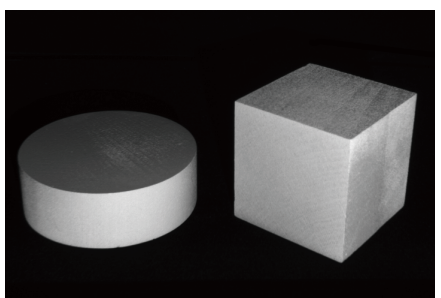

Cube and Disc

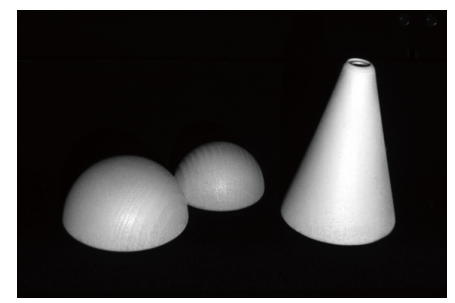

Cone and Spheres

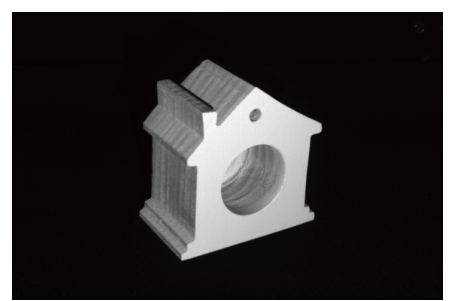

Toy House

\begin{tabular}{llllllllll}
\hline Technique & Multi & Comp 8 & Comp 16 & Multi & Comp 8 & Comp 16 & Multi & Comp 8 & Comp 16 \\
\hline Captured points & 109347 & 108306 & 109034 & 46749 & 46263 & 46505 & 37457 & 36880 & 37133 \\
Average deviation & 0.025 & 0.089 & 0.063 & 0.030 & 0.085 & 0.067 & 0.036 & 0.088 & 0.068 \\
Average distance & - & 32.00 & 25.09 & - & 21.15 & 16.19 & - & 23.26 & 20.87 \\
\hline
\end{tabular}

Figure 5. Comparison between reconstructions obtained with the Multi-Period Phase Shift and the Compound Phase Coding on several test objects. Compound Phase Coding has been tested using both 8 and 16 samples. Multi-Period Phase Shift has been tested with 34 samples in order to obtain the best quality. Distances are in microns and objects are 5 to $10 \mathrm{~cm}$ wide.

\subsection{Generic objects measurements}

In this set of experiments we measured the surface of several generic scenes built with simple wooden objects in a volume of about $200 \mathrm{~mm}$ of diameter in each direction. The set of objects was selected to maximize the range of surface orientations and conditions. Again we compared our results with those obtained by the Multi-Period approach: in this case we take the Multi-Period results as a direct comparison, as no ground-truth or knowledge of the surface of the objects (which are hand-made) is available. Compound Phase Coding was tested both with 8 and 16 projected patterns. Since we projected signals with periods of respectively 9, 11 and 13 pixels Multi-Period Phase Shift was tested with 34 samples. For each experiment we evaluated three quantities, namely:

- The number of points acquired. That is the number of surface points that pass both the consistency and the quality check. The first verifies that the phase vector is consistent with the code assigned to the point, the latter ensures that the phase difference vector contains only integer values (as expected with respect to constraint 2). Specifically, for these experiments we rejected points associated to phase difference vectors where at least one entry deviates from the nearest integer by more than 0.2 ;

- The average deviation. That is the average distance from each entry in the phase difference vector and the nearest integer. Since each element of the phase difference vector should be integer a lower value suggests a higher quality in the measurement obtained. This parameter has been calculated over all the points obtained, even those filtered by the consistency and quality checks;
- The average distance. That is the average of the absolute distances between points obtained by the MultiPeriod and Compound techniques. Note that this measure is possible because we implemented both algorithms in a way that allowed us to produce depth maps that are exactly overlapped along the $\mathrm{x}$ and $\mathrm{y}$ axis and differ only for the depth values assigned along the $\mathrm{z}$ axis.

In Figure 5 we report the values of those three quantities in different scenes. In general, the number of points acquired by the Compound Phase Coding with only 8 patterns is slightly smaller than the number of valid points given by the Multi-Period technique. This distance, while small, is almost completely eliminated when using 16 patterns. The average deviation resulting by applying our technique is always slightly higher, nevertheless it should be remarked that those values are all quite small: in fact, for any practical purpose a deviation smaller than 0.1 can always be associated with a valid measured point. Finally, the average distances between the two techniques also assess the suitability of our technique as a fast replacement for traditional Multi-Period: in fact, the results obtained are generally compatible with the measurement error estimated in the experiments done in section 3.2, a result that should be expected since both techniques are subject to measurement errors. It should also be noted that the higher average distance obtained with the scene "Cube and Disc" is probably due to the presence of sharp edges and of two large surfaces at a grazing angle with both the cameras and the light source. Conversely, the higher smoothness offered by the scene "Cone and Spheres" allows for a more precise reconstruction.

In Figure 6 we show a more qualitative example of the difference in accuracy of reconstruction between our method and a complete Multi-Period phase shift. In this experiment we used the less accurate instance of Compound 

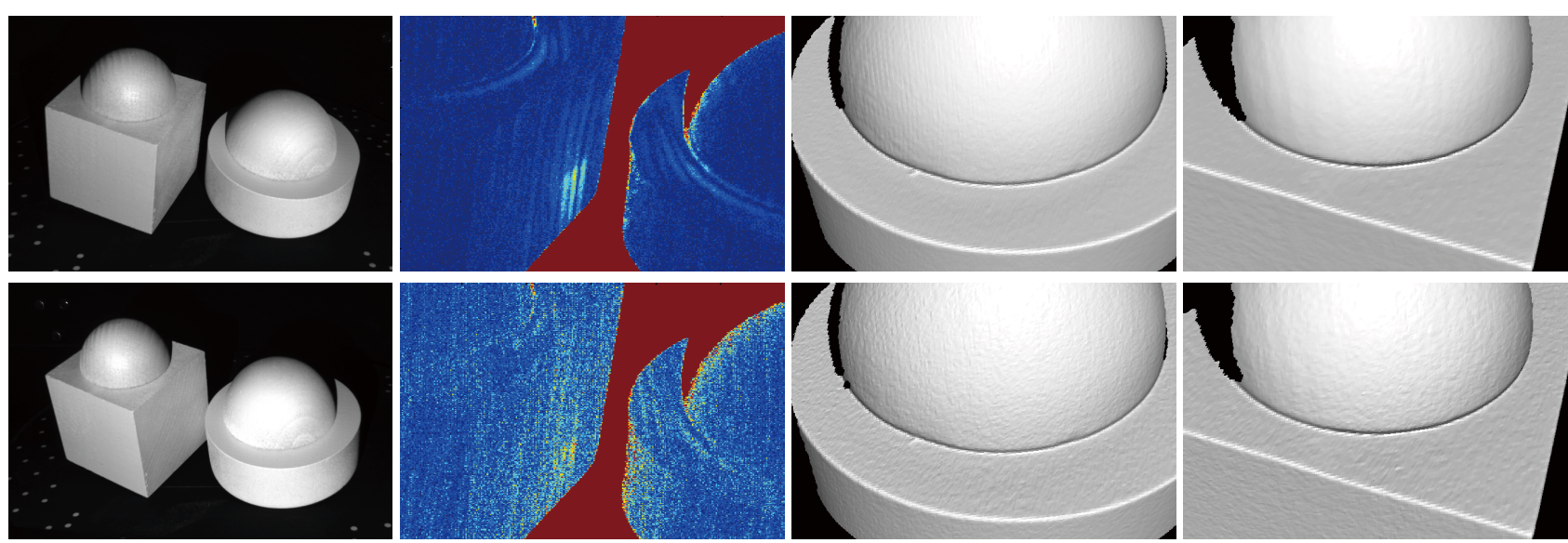

Figure 6. A surface reconstruction example complete with deviation assessment. In the first colum, the object as viewed by the left and right camera. In the second column, the deviance maps of Multi-Period Phase Shift (top) and Compound Phase Coding (bottom). In the last colums, two respective close-ups of the reconstructed surfaces.

Phase Coding, thus only 8 patterns were projected. In the first column we reproduced the scene viewed from the left and right camera. In the second column we calculated a map of the maximum deviation from integer values of the phase difference vectors of the Multi-period method (top) and the Compound method (bottom). In these maps we used the standard Matlab color scale, thus a full blue pixel indicates a low deviation and a bright red pixel a maximum deviation (in this case 0.5). Uncoded pixels are represented in dark red. Both techniques exhibit a fairly low deviation, with high values mainly on the borders, which is due to the camera integration of the projected signal and the background on boundary pixels. The other high deviation area on the side of the cube is probably due to inter-reflection between the two objects.

The last two columns show a close-up of the surfaces reconstructed with both methods. In general the surface reconstructed with the Compound technique is a bit more noisy, but the overall level of detail is maintained, even with details at a relatively high frequency being clearly visible on both reconstructions: for instance, in the close-up of the disc object (third column) a very small bulge is visible in both the reconstructions in proximity of the lower rim of the hemisphere.

Finally, in Figure 7, we compare the reconstructed phases obtained after the application of the Discrete Fourier Transform to the captured structured light images before and after the coding step. As in the previous experiments we choose periods of 9,10 and 13 pixels and we projected 34 patterns for the Multi-Period method and 8 pattern for our approach. In the first column we show respectively the complete coding for the Multi-Period (top) and for the Compound (middle) methods. In order to highlight the differences between the two codings, we show in the third line of this column the difference image between them: as ex- pected they are almost identical and the only slight differences are found in boundary regions, where the measurements are less precise.

In the other two columns we show the phase related to two different signals calculated by the two techniques (we show only two signals for space reasons). For the purpose of a simple comparison between them, we plotted a magnified graph of the small red lines in the phase images. In those graphs the red line represents the phase extracted by the Multi-Period method and the blue line the phase extracted by our technique. It can be seen that the values obtained are very similar, and that they diverge by a very small amount only in proximity of critical areas such as the interface between the disc and the hemisphere.

\section{Conclusions}

In this paper we have proposed a novel compound phase coding technique that is able to perform a complete and accurate surface reconstruction requiring the projection of as few as 8 patterns. This is obtained by encoding multiple phase information in a single pattern sequence as phases of sinusoidal signals at integer frequencies, thus encoding and decoding the compound signal using standard Fourier analysis. The comparison with another well known technique assesses the ability of the approach to obtain accurate reconstruction even with very few patterns. In addition, the time/quality trade-off can be easily controlled by adding more patterns. In fact, we show that measurement error decreases consistently by adding more information, to the point that our method reaches the performance of other state-of-the-art approaches when feeded with a comparable quantity of data. 

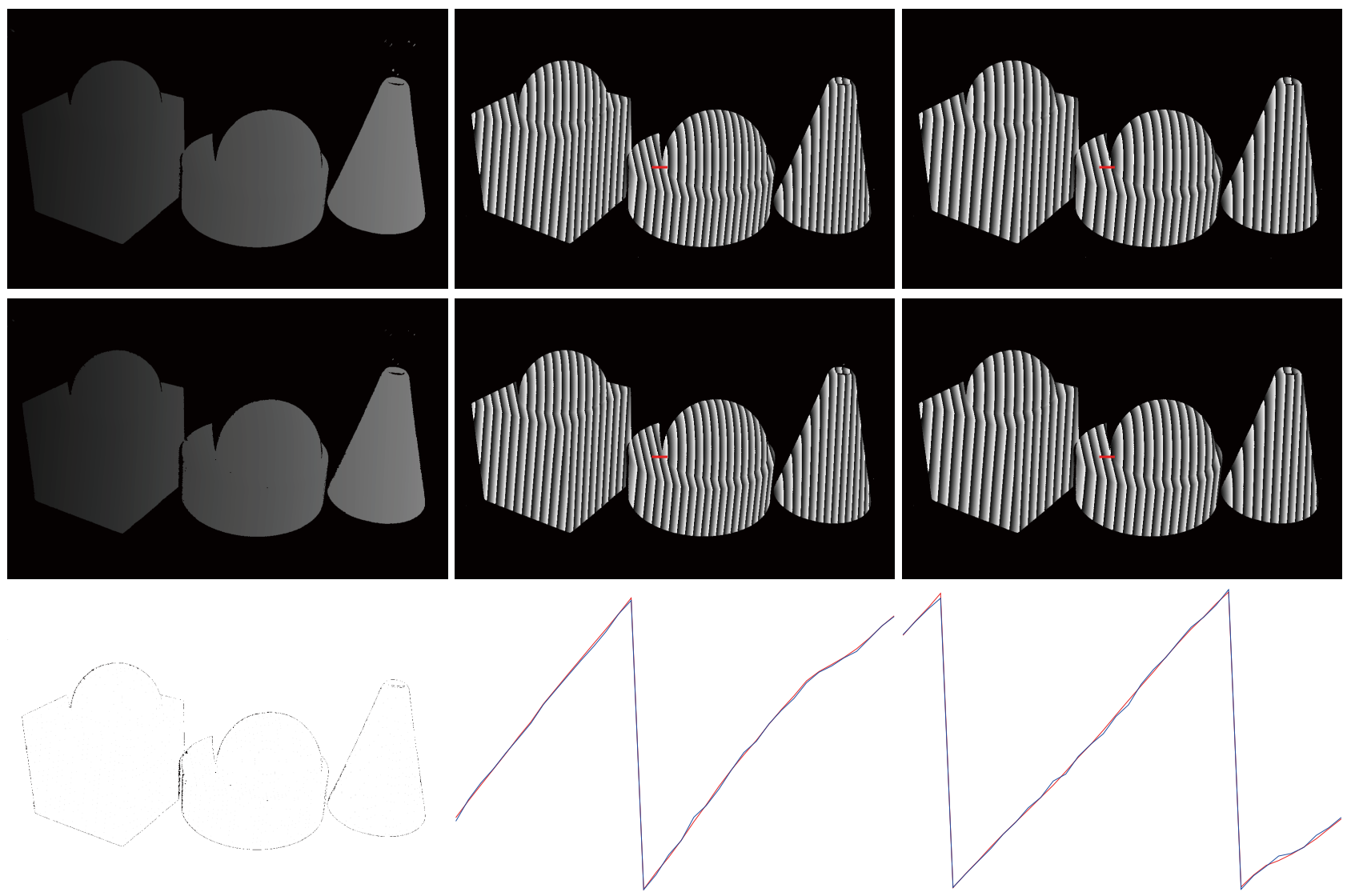

Figure 7. A comparison of the coding and of the phase values extracted by our technique and by Multi-Period phase shift. In the first colum we show the final codings and their difference. In the other columns we display the respective phase images for two reconstructed signals. The two magnified plots in the last row correspond to the small red segment. Note that the obtained phases are very similar even in the proximity of critical areas.

\section{References}

[1] G. Bradski and A. Kaehler. Learning OpenCV: Computer Vision with the OpenCV Library. O'Reilly Media, Inc., 1st edition, October 2008.

[2] W. Chen, H. Yang, X. Su, and S. Tan. Error caused by sampling in fourier transform profilometry. Optical Engineering, 38(6):1029-1034, 1999.

[3] C. Guan, L. Hassebrook, and D. Lau. Composite structured light pattern for three-dimensional video. Opt. Express, 11(5):406-417, 2003.

[4] E. M. M. Joan Batlle and J. Salvi. Recent progress in coded structured light as a technique to solve the correspondence problem: a survey. Pattern Recognition, 31(7):963-982, 1998.

[5] J. P. Joaquim Salvi and J. Batlle. Pattern codification strategies in structured light systems. Pattern Recognition, 37:827-849, 2004

[6] E. Lilienblum and B. Michaelis. Optical $3 d$ surface reconstruction by a multi-period phase shift method. JCP, 2(2):73-83, 2007.

[7] D. Scharstein and R. Szeliski. A taxonomy and evaluation of dense two-frame stereo correspondence algorithm. Int. J. of
Comp. Vision, 47(1):7-42, 2002.

[8] V. Srinivasan, H. C. Liu, and M. Halioua. Automated phasemeasuring profilometry: a phase mapping approach. Appl. Opt., 24(2):185-188, 1985.

[9] X. Su and W. Chen. Fourier transform profilometry: a review. Optics and Lasers in Engineering, 35:263-284, May 2001.

[10] Y. Surrel. Design of algorithms for phase measurements by the use of phase stepping. Appl. Opt., 35(1):51-60, 1996.

[11] M. Takeda and K. Mutoh. Fourier transform profilometry for the automatic measurement of 3-d object shapes. Appl. Opt., 22(24):3977-3982, 1983.

[12] C. Xiaobo, X. J. tong, J. Tao, and J. Ye. Research and development of an accurate $3 d$ shape measurement system based on fringe projection: Model analysis and performance evaluation. Precision Engineering, 32(3):215 - 221, 2008.

[13] H.-M. Yue, X.-Y. Su, and Y.-Z. Liu. Fourier transform profilometry based on composite structured light pattern. Optics Laser Technology, 39:1170-1175, Sept. 2007.

[14] J. Zhong and Y. Zhang. Absolute phase-measurement technique based on number theory in multifrequency grating projection profilometry. Appl. Opt., 40(4):492-500, 2001. 